\title{
Metformin inhibits expression of the proinflammatory biomarker inducible nitric oxide synthase in hepatocytes
}

\section{Richi Nakatake $^{1 *}$, Hiroya Iida ${ }^{2}$, Morihiko Ishizaki ${ }^{1}$, Kosuke Matsui ${ }^{1}$, Yusuke Nakamura ${ }^{1}$, Masaki Kaibori' $^{1}$, Mikio Nishizawa ${ }^{3}$ and Tadayoshi Okumura ${ }^{1,4}$}

${ }^{1}$ Department of Surgery, Kansai Medical University, Hirakata, Osaka, Japan; ${ }^{2}$ Department of Surgery, Shiga University of Medical Science, Otsu, Shiga, Japan; ${ }^{3}$ Department of Biomedical Sciences, College of Life Sciences, Ritsumeikan University, Kusatsu, Shiga, Japan; ${ }^{4}$ Research Organization of Science and Technology, Ritsumeikan University, Kusatsu, Shiga, Japan

Corresponding author: Richi Nakatake, M.D. and Ph.D., Department of Surgery, Kansai Medical University, 2-5-1 Shinmachi, Hirakata, Osaka, 573-1010, Japan

Submission Date: December 26th, 2017, Acceptance Date: March 27 ${ }^{\text {th }}$, 2018, Publication Date: March $31^{\text {st }}, 2018$

Citation: Nakatake R., Iida H., Ishizaki M., Matsui K., Nakamura Y., Kaibori M., Nishizawa M., Okumura T., Metformin inhibits expression of the proinflammatory biomarker inducible nitric oxide synthase in hepatocytes. Functional Foods in Health and Disease 2018; 8(3): 175-192. DOI: https://doi.org/10.31989/ffhd.v8i3.423

\begin{abstract}
Background: Metformin is used to treat patients with type II diabetes. However, there are few scientific reports on its anti-inflammatory effects. In the inflamed liver, proinflammatory cytokines stimulate liver cells, followed by inducible nitric oxide synthase (iNOS) expression. Excessive NO levels produced by iNOS have been implicated as a factor in liver injury. As a result, it is essential to inhibit iNOS induction to prevent liver injury.
\end{abstract}

Objective: This study aimed to investigate liver protective effects of metformin by examining interleukin (IL)-1 $\beta$-stimulated hepatocytes.

Methods: Primary cultured rat hepatocytes were treated with interleukin (IL)-1 $\beta$ in the presence or absence of metformin. iNOS induction and its signaling pathway were analyzed. 
Results: Metformin decreased iNOS protein and mRNA expression, resulting in the inhibition of hepatic NO production. Metformin also reduced tumor necrosis factor (TNF)- $\alpha$ and IL-6 mRNA expression. Metformin inhibited an essential signaling pathway for iNOS induction, type I IL-1 receptor upregulation. Transfection experiments revealed that metformin reduced iNOS mRNA levels through both promoter transactivation and mRNA stabilization. Delayed metformin administration after IL-1 $\beta$ addition also inhibited iNOS induction.

Conclusion: Metformin affects the induction of inflammatory mediators including iNOS and TNF- $\alpha$, demonstrating its therapeutic potential for organ injuries, including the liver.

Keywords: metformin, inducible nitric oxide synthase, liver injury, primary cultured hepatocytes, type I interleukin-1 receptor, tumor necrosis factor- $\alpha$

\section{INTRODUCTION}

Metformin is generally one of the most effective therapeutics for the treatment of type 2 diabetes because it specifically reduces hepatic gluconeogenesis without increasing insulin secretion, inducing weight gain, or posing a risk of hypoglycemia [1]. The anti-diabetic action of metformin depends on the activation of AMP-activated protein kinase (AMPK), which contributes to a reduction in hepatic gluconeogenesis and an increase in glucose uptake in skeletal muscles [2].

Insulin resistance is one of the major pathophysiological features which is implicated in nonalcoholic fatty liver disease (NAFLD) [3,4], contributing to both the initiation of the disease and progression to advanced forms of NAFLD. NAFLD is one of the most prevalent liver diseases world-wide and it is an increasingly frequent cause of cirrhosis $[5,6]$. The disease encompasses a wide spectrum of liver damage ranging from simple hepatic steatosis and non-alcoholic steatohepatitis (NASH) to liver cirrhosis.

In hepatic disorders, inflammatory cells such as macrophages gather around hepatic stellate (Kupffer) cells and discharge a variety of cytokines. During inflammation, proinflammatory cytokines and nitric oxide (NO), which is produced by inducible nitric oxide synthase (iNOS) in Kupffer cells and hepatocytes, play an important role as factors in liver injury [7]. iNOS expression was enhanced in the liver after feeding with a high fat diet [8,9]. Given the critical role of iNOS in the development of liver fibrosis, long-term inhibition of iNOS may have therapeutic benefits in liver steatotis [10].

In animal liver injury models caused by various insults, such as ischemia-reperfusion, partial hepatectomy, and endotoxin shock, we discovered that drugs [11-15] demonstrating liverprotective effects inhibited the induction of iNOS and NO production, and reduced production of various inflammatory mediators, such as tumor necrosis factor (TNF)- $\alpha$, interleukin (IL)-1 $\beta$, IL-6, 
and cytokine-induced neutrophil chemoattractant (CINC)-1 (human IL-8 analogue) [11-15]. Additionally, these clinical drugs [13,16,17] also inhibited the induction of iNOS and NO production in primary cultures of rat hepatocytes. Therefore, experiments using cultured hepatocytes revealed that preventing iNOS induction and NO production is an indicator of liver protection [10].

It has recently been demonstrated that metformin alleviates fulminant liver injury in lipopolysaccharide (LPS)/D-galactosamine-challenged mice [18]. Metformin-mediated amelioration of LPS/D-galactosamine induced hepatitis in mice, which correlated with reduced iNOS expression and NO production in macrophages $[19,20]$, although the mechanisms involved in the action of metformin are unclear.

In the present study, we examined whether metformin affects the induction of iNOS in IL-1 $\beta$ stimulated hepatocytes and the mechanisms involved in the action of metformin.

\section{MATERIALS AND METHODS}

\section{Materials}

Metformin (Wako Pure Chemicals, Osaka, Japan) was dissolved in Williams' medium E (WE) and vortexed for $10 \mathrm{~min}$ at room temperature. The supernatant was filter-sterilized with a $0.45-\mu \mathrm{m}$ membrane filter (Millipore, Billerica, MA, USA) before use in experiments. Recombinant human IL-1 $\beta\left(2 \times 10^{7} \mathrm{U} / \mathrm{mg}\right.$ protein) was purchased from My Bio Source (San Diego, CA, USA). Male Wistar rats (200-250 g and 6-7 weeks old) were purchased from Charles River (Tokyo, Japan), kept at $22^{\circ} \mathrm{C}$ under a $12: 12 \mathrm{~h}$ light:dark cycle, and received food and water ad libitum. All experiments on rats were performed in accordance with the Guidelines for the Care and Use of Laboratory Animals of the National Institutes of Health. The experiments were approved by the Animal Care Committee of Kansai Medical University.

\section{Primary hepatocyte culture}

Hepatocytes were isolated from rats by perfusion with collagenase (Wako Pure Chemicals) [21]. Isolated hepatocytes were suspended in culture medium at $6 \times 10^{5}$ cells $/ \mathrm{mL}$, seeded into $35-\mathrm{mm}$ plastic dishes ( $2 \mathrm{~mL} /$ dish; Falcon Plastic, Oxnard, CA, USA), and cultured at $37^{\circ} \mathrm{C}$ in a $\mathrm{CO}_{2}$ incubator under a humidified atmosphere of $5 \% \mathrm{CO}_{2}$ in air. The culture medium was WE supplemented with 10\% newborn calf serum, $10 \mathrm{nM}$ insulin, and $10 \mathrm{nM}$ dexamethasone. After 5 $\mathrm{h}$, the medium was replaced with fresh serum and hormone-free WE, and the cells were cultured overnight before use in experiments. The number of cells attached to the dishes was calculated by counting the number of nuclei [22] and using a ratio of $1.37 \pm 0.04$ nuclei/cell (mean $\pm \mathrm{SE}, \mathrm{n}=7$ experiments). 


\section{Treatment of cells with metformin}

On day 1, the cells were washed with fresh serum- and hormone-free WE, and incubated with IL$1 \beta(1 \mathrm{nM})$ in the same medium in the presence or absence of metformin. The doses of metformin used are indicated in the appropriate Figures and their legends.

\section{Determination of $\mathrm{NO}$ production and lactate dehydrogenase activity}

The culture medium was used to measure nitrite (a stable metabolite of NO) levels, which reflects NO production, using the Griess method [23]. Culture medium was also used for measurements of lactate dehydrogenase (LDH) activity to reflect cell viability using a commercial kit (Roche Diagnostics, Mannheim, Germany).

\section{Western blot analysis}

Total cell lysates were obtained from cultured cells as described previously, with minor modifications [16]. Briefly, cells $\left(1 \times 10^{6}\right.$ cells/35 mm dish) were lysed with sample buffer for sodium dodecyl sulfate-polyacrylamide gel electrophoresis (SDS-PAGE) (final: $125 \mathrm{mM}$ Tris-HCl, pH 6.8; containing 5\% glycerol, 2\% SDS, and 1\% 2-mercaptoethanol), subjected to SDS-PAGE, and electroblotted onto a polyvinylidene difluoride membrane (Bio-Rad, Hercules, CA, USA). Immunostaining was performed using primary antibodies against mouse iNOS (Affinity BioReagents, Golden, CO, USA), human IkB $\alpha$, mouse type I IL-1 receptor (IL-1RI) (Santa Cruz Biotechnology, Santa Cruz, CA, USA), and rat $\beta$-tubulin (internal control; Clone TUB2.1; Sigma Chemical Co., St. Louis, MO, USA). This was followed by visualization with an enhanced chemiluminescence blotting detection reagent (GE Healthcare Biosciences Corp., Piscataway, NJ, USA).

For Akt, total cell lysates prepared from $100-\mathrm{mm}$ dishes $\left(5 \times 10^{6}\right.$ cells/dish $)$ were pre-cleared with protein A (Sigma Chemical Co.) and then mixed with a mouse monoclonal antibody against human Akt1 (Akt5G3; Cell Signaling, Beverly, MA, USA) and protein G-Sepharose (Pharmacia LKB Biotech, Uppsala, Sweden). After incubation overnight at $4^{\circ} \mathrm{C}$, immunocomplexes were centrifuged $(16,000 \times g$ for $5 \mathrm{~min})$. The beads were washed with solubilizing buffer, dissolved in SDS-PAGE sample buffer, and analyzed by western blotting using rabbit polyclonal antibodies against human Akt and phospho-Akt (Ser473) (Cell Signaling) as primary antibodies. For p65, nuclear extracts were immunoprecipitated with an anti-p65 antibody (H286; Santa Cruz Biotechnology). The bands were analyzed by western blotting using an antibody against human NF-кB p65 (BD Transduction Laboratories, Lexington, KY, USA).

\section{Reverse transcriptase-polymerase chain reaction (RT-PCR)}

Total RNA was extracted from cultured hepatocytes using the guanidinium-phenol-chloroform method [24]. For strand-specific RT-PCR analysis, cDNA was synthesized from total RNA using strand-specific primers, and step-down PCR was performed using PC708 (Astec, Fukuoka, Japan), 
as previously described [25], with minor modifications. For iNOS (257 bp), TNF- $\alpha$ (275 bp), IL6 (286 bp), IL-10 (245 bp), CINC-1 (231 bp), IL-1RI (327 bp), and elongation factor-1 $\alpha$ (EF; internal control) (335 bp) mRNA, an oligo(dT) primer was used for RT and the indicated primers sets were used for PCR (Table 1). For the antisense transcript of iNOS (211 bp), the sense primer (5'-TGCCCCTCCCCCACATTCTCT-3') was used for RT and the indicated primer set was used for PCR (Table 1). These mRNAs and antisense transcript levels were measured with real-time PCR using the Rotor-Gene Q 2plex HRM (Qiagen, Tokyo, Japan). The Rotor-Gene SYBR Green PCR Kit (Qiagen) was included in the reaction mixture, and the following touchdown protocol was applied: 1 cycle at $95^{\circ} \mathrm{C}$ for 5 min and 45 cycles at $95^{\circ} \mathrm{C}$ for $5 \mathrm{~s}$ and $60^{\circ} \mathrm{C}$ for $10 \mathrm{~s}$. The cDNAs for the rat iNOS mRNA and antisense transcript were deposited in the DNA Data Bank of Japan/European Bioinformatics Institute (DDBJ/EMBL)/GenBank under the accession numbers $\mathrm{AB} 250951$ and $\mathrm{AB} 250952$ respectively.

\section{Electrophoretic mobility shift assay (EMSA)}

Nuclear extracts were prepared and an electrophoretic mobility shift assay was performed as previously described [26]. Briefly, nuclear extracts from hepatocytes $(4 \mu \mathrm{g})$ were mixed with $1 \mu \mathrm{g}$ of poly $(\mathrm{dI}-\mathrm{dC})$ and a probe for $20 \mathrm{~min}$ at room temperature (total mixture, $15 \mu \mathrm{l})$. To prepare the double-stranded DNA probe, annealed oligonucleotides harboring a $\kappa \mathrm{B}$ site (5'AGTTGAGGGGACTTTCCCAGGC-3'; only the sense strand is shown) were labelled with [ $\gamma$ ${ }^{32} \mathrm{P}$ ]-adenosine-5'-triphosphate (ATP; DuPont-New England Nuclear Japan, Tokyo, Japan) and T4 polynucleotide kinase (Takara Bio Inc., Shiga, Japan). Samples were resolved on a 4.8\% polyacrylamide gel, followed by drying and autoradiography. Protein concentrations were measured using the Bradford method [27] with a binding assay kit (Bio-Rad) using bovine serum albumin as a standard.

\section{Transfection and luciferase assay}

Transfection of cultured hepatocytes was performed as previously described [28]. Briefly, hepatocytes were cultured at $4 \times 10^{5}$ cells/dish $(35 \times 10 \mathrm{~mm})$ in WE supplemented with serum, dexamethasone, and insulin for $7 \mathrm{~h}$, before being subjected to magnet-assisted transfection (MATra). The reporter plasmid pRiNOS-Luc-SVpA or pRiNOS-Luc-3'UTR (1 $\mu \mathrm{g})$ and the CMV promoter-driven $\beta$-galactosidase plasmid pCMV-LacZ (1 ng) as an internal control were mixed with MATra-A reagent ( $1 \mu \mathrm{l}$; IBA GmbH, Göttingen, Germany). After incubation for 15 min on a magnetic plate at room temperature, the medium was replaced with fresh WE containing serum. Cells were cultured overnight, and then treated with IL-1 $\beta$ in the presence or absence of metformin. Luciferase and $\beta$-galactosidase activities of cell extracts were measured using PicaGene (Wako Pure Chemicals) and Beta-Glo (Promega, Wisconsin, USA) kits respectively. 


\section{Statistical analysis}

The results shown are representative of three to four independent experiments yielding similar findings. All data are expressed as the mean \pm SD. Differences were analyzed by the BonferroniDunn test, and a value of $\mathrm{P}<0.05$ was considered to indicate a significant difference.

Table 1. Primers and nucleotide sequences

\begin{tabular}{|c|c|c|}
\hline $\begin{array}{l}\text { Primer } \\
\text { name }\end{array}$ & Nucleotide sequence & \\
\hline \multirow[t]{2}{*}{ iNOS F/R } & CCAACCTGCAGGTCTTCGATG & $3 ' / 5$ ' \\
\hline & \multicolumn{2}{|l|}{ GTCGATGCACAACTGGGTGAAC 3' } \\
\hline as iNOS & CCTTTGCCTCATACTTCCTCAGA & $3^{\prime} / 5^{\prime}$ \\
\hline $\mathrm{F} / \mathrm{R}$ & \multicolumn{2}{|l|}{ ATCTTCATCAAGGAATTATACACGG 3' } \\
\hline \multirow[t]{2}{*}{$\mathrm{TNF}-\alpha \mathrm{F} / \mathrm{R}$} & TCCCAACAAGGAGGAGAAGTTCC & $3^{\prime} / 5^{\prime}$ \\
\hline & \multicolumn{2}{|l|}{ GGCAGCCTTGTCCCTTGAAGAGA 3' } \\
\hline \multirow[t]{2}{*}{ IL-6 F/R } & GAGAAAAGAGTTGTGCAATGGCA & $3{ }^{\prime} / 5^{\prime}$ \\
\hline & \multicolumn{2}{|l|}{ TGAGTCTTTTATCTCTTGTTTGAAG 3' } \\
\hline \multirow[t]{2}{*}{ CINC-1 F/R } & GCCAAGCCACAGGGGCGCCCGT & $3^{\prime} / 5^{\prime}$ \\
\hline & \multicolumn{2}{|l|}{ ACTTGGGGACACCCTTTAGCATC 3' } \\
\hline \multirow[t]{2}{*}{ IL-1RI F/R } & \multicolumn{2}{|l|}{ 5'-CGAAGACTATCAGTTTTTGGAAC-3'/5'- } \\
\hline & \multicolumn{2}{|l|}{ GTCTTTCCATCTGAAGCTTTTGG-3' } \\
\hline \multirow[t]{2}{*}{$\mathrm{IL}-10 \mathrm{~F} / \mathrm{R}$} & GCAGGACTTTAAGGGTTACTTGG & $3^{\prime} / 5^{\prime}$ \\
\hline & \multicolumn{2}{|l|}{ CCTTTGTCTTGGAGCTTATTAAA 3' } \\
\hline \multirow[t]{2}{*}{$\mathrm{EF} F / \mathrm{R}$} & TCTGGTTGGAATGGTGACAACATGC & $3 ' / 5^{\prime}$ \\
\hline & \multicolumn{2}{|l|}{ CCAGGAAGAGCTTCACTCAAAGCTT 3' } \\
\hline
\end{tabular}

iNOS, inducible nitric oxide synthase; as, antisense transcript; TNF- $\alpha$, tumor necrosis factoralpha; IL-6, interleukin-6; CINC-1, cytokine-induced neutrophil chemoattractant-1; IL-1R1, type I IL-1 receptor; IL-10, interleukin-10; EF, elongation factor-1 $\alpha$; F/R, forward/reverse

\section{RESULTS}

\section{Metformin inhibits NO production and iNOS induction.}

The proinflammatory cytokine IL-1 $\beta$ stimulated iNOS induction, which was followed by production of NO in primary cultured rat hepatocytes. Simultaneous addition of metformin and IL-1 $\beta$ reduced the levels of nitrite (a stable metabolite of NO) in a time and dose-dependent manner in the culture medium (Fig. 1A and 1B). Metformin demonstrated more than 90\% inhibition at 500 $\mu \mathrm{g} / \mathrm{ml}$. Metformin had no cellular cytotoxicity at the various concentrations, as evaluated by the release of LDH into the culture medium (Fig. 2) and Trypan blue exclusion in hepatocytes (data not shown). Western blotting analysis revealed that metformin dose-dependently reduced iNOS 
protein expression, showing its IC50 at $200 \mu \mathrm{g} / \mathrm{ml}$ (Fig. 1B). RT-PCR analysis revealed that metformin decreased iNOS mRNA expression in a time-dependent manner (Fig. 1C). These results suggested that metformin inhibited the induction of iNOS gene expression at the transcriptional and/or post-transcriptional level
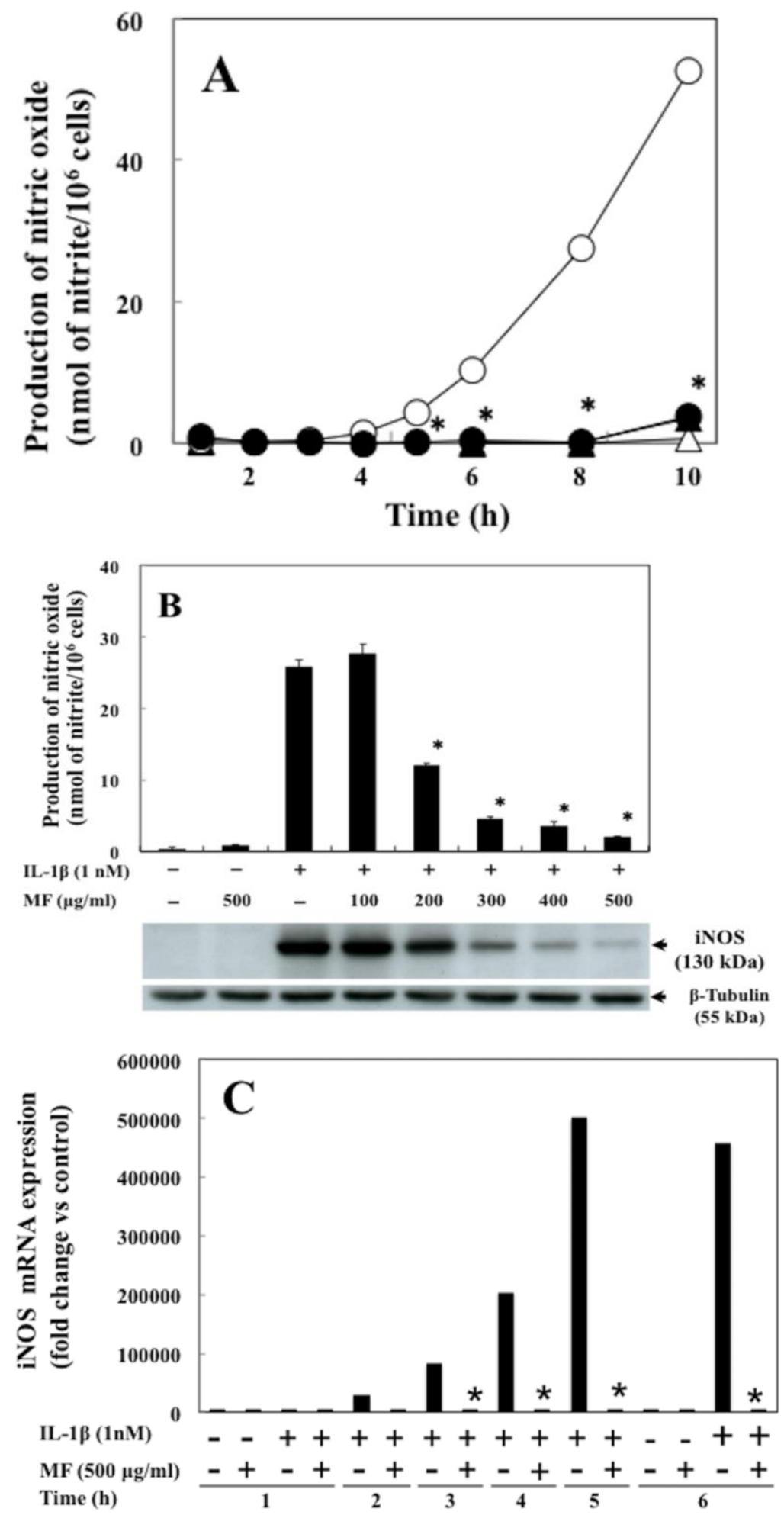

Figure 1. Effects of metformin on induction of NO production and iNOS in IL-1 $\beta$-stimulated hepatocytes. Cultured hepatocytes 
were treated with interleukin (IL)-1 $\beta(1 \mathrm{nM})$ in the presence or absence of metformin $(100-500 \mu \mathrm{g} / \mathrm{ml})$. (A) Effect of metformin $(500 \mu \mathrm{g} / \mathrm{ml})$ treatment for the indicated times on nitric oxide (NO) production (IL-1 $\beta$, open circles; IL-1 $\beta+$ metformin, filled circles; metformin, filled triangles; controls [without IL-1 $\beta$ and metformin], open triangles). (B) Effects of treatment with various doses of metformin (100-500 $\mu \mathrm{g} / \mathrm{ml})$ for $8 \mathrm{~h}$ on NO production (upper panel) and inducible nitric oxide synthase (iNOS) protein levels (medium). Nitrite levels were measured in the culture medium. Data are presented as the mean \pm SD for $n=3$ dishes/point; ${ }^{*} \mathrm{P}<0.05$ versus IL-1 $\beta$ alone. In the western blot panels, cell lysates ( $20 \mu \mathrm{g}$ of protein) were subjected to sodium dodecyl sulfate-polyacrylamide gel electrophoresis in a $7.5 \%$ gel, and immunoblotted with an anti-iNOS or anti- $\beta$-tubulin antibody. (C) Effects of metformin $(500 \mu \mathrm{g} / \mathrm{ml})$ treatment for the indicated times on iNOS mRNA expression. Total RNA was analyzed by strand-specific RT-PCR to detect iNOS mRNA, using EF mRNA as an internal control.

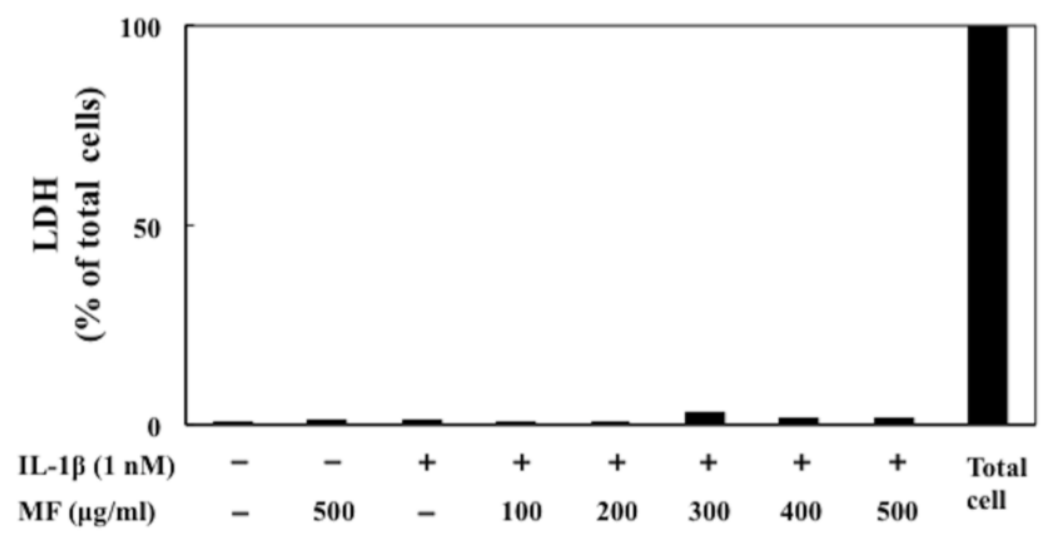

Figure 2. Effects of metformin on cellular cytotoxicity. Cells were treated with IL-1 $\beta(1 \mathrm{nM})$ in the presence or absence of metformin $(100-500 \mu \mathrm{g} / \mathrm{ml})$ for $8 \mathrm{~h}$. Lactate dehydrogenase (LDH) activity was measured in the culture medium (data are presented as the mean $\pm \mathrm{SD}, \mathrm{n}=3$ dishes/point).

\section{Metformin affects mRNA expression of proinflammatory cytokines.}

The expression of other mRNAs was examined. IL-1 $\beta$ increased the levels of proinflammatory cytokine expression, such as TNF- $\alpha$, CINC-1, and IL- 6 , and metformin decreased TNF- $\alpha$, CINC1, and IL-6 mRNA levels (Fig. 3A, 3B, and 3C). However, metformin increased the mRNA levels of the anti-inflammatory cytokine IL-10, while IL-1 $\beta$ had no effects on IL-10 mRNA levels (Fig. 3D).

\section{Metformin decreases iNOS mRNA synthesis and stabilization.}

We examined the mechanisms that are involved in inhibiting iNOS induction. iNOS mRNA expression is regulated by iNOS promoter transactivation with transcription factors, such as NF$\kappa \mathrm{B}$, and by post-transcriptional modifications, such as mRNA stabilization [29]. Therefore, we performed transfection experiments using constructs containing firefly luciferase controlled by the iNOS promoter (pRiNOS-Luc-SVpA and pRiNOS-Luc-3'UTR; Fig. 4A), which detects iNOS promoter transactivation (mRNA synthesis) and mRNA stabilization. IL-1 $\beta$ increased the luciferase activity of these constructs, and these effects were significantly inhibited by metformin (Fig. 4B and 4C). Additionally, iNOS antisense transcript (asRNA) analysis using real-time RT- 
PCR revealed that IL-1 $\beta$ increased iNOS asRNA expression in a time-dependent manner and that metformin markedly inhibited this effect (Fig. 4D).
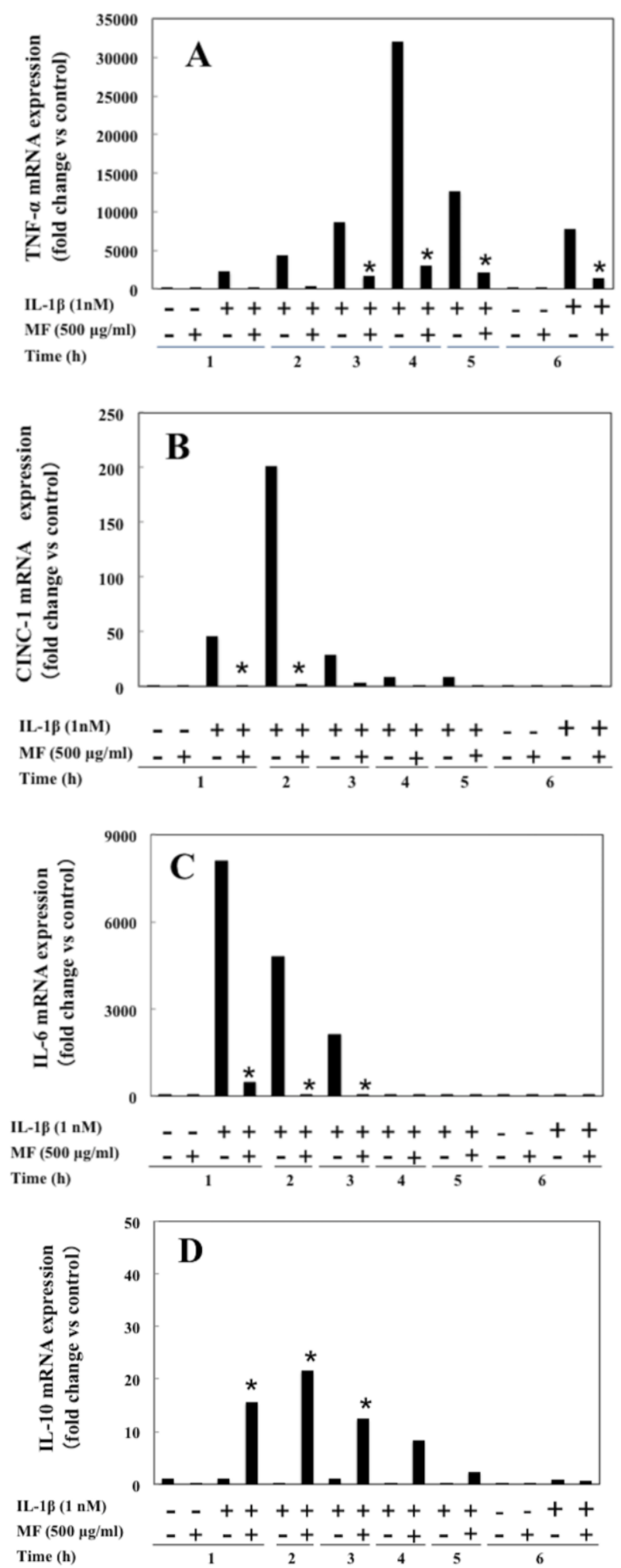

Figure 3. Effects of metformin on TNF- $\alpha$, CINC-1, IL-6, and IL-10 mRNA expression. Cells were treated with IL-1 $\beta(1 \mathrm{nM})$ in the presence or absence of metformin $(500 \mu \mathrm{g} / \mathrm{ml})$ for the indicated times. Total RNA was analyzed using strand-specific RT-PCR to detect (A) TNF- $\alpha$, (B) CINC-1, (C) IL-6, and (D) IL-10 using EF mRNA as an internal control. 


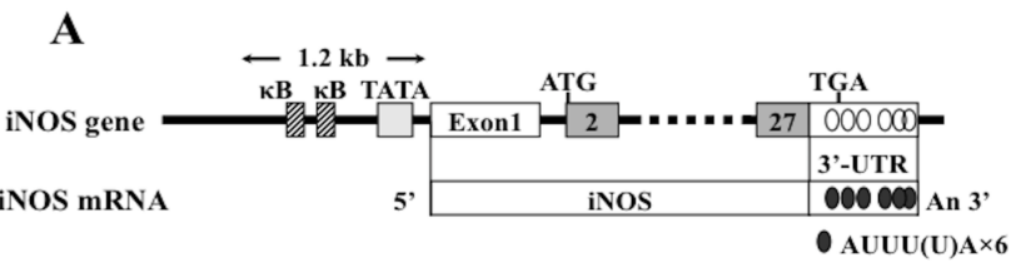

pRiNOS-Luc-SVpA (mRNA synthesis)

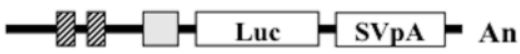

pRiNOS-Luc-3'UTR (mRNA stabilization)
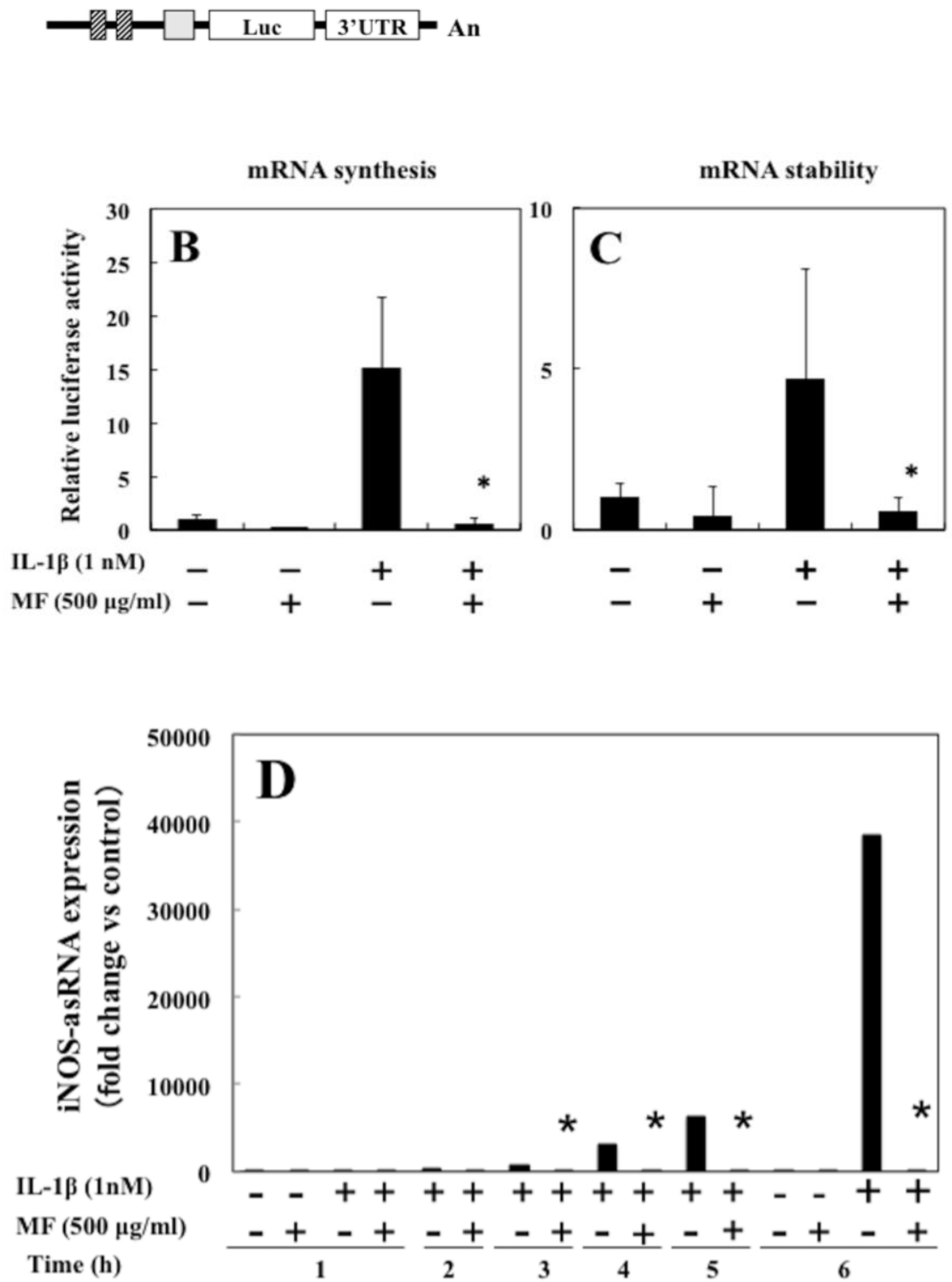

Figure 4. Effects of metformin on iNOS promoter transactivation and iNOS gene antisense transcript expression. (A) Schematic representation of the iNOS gene promoter region. Two reporter constructs are shown beneath the iNOS gene and mRNA. The constructs consist of the rat iNOS promoter $(1.2 \mathrm{~kb})$, a luciferase gene, and the SV40 poly(A) region (pRiNOS-Luc-SVpA) or iNOS 3'-UTR (pRiNOS-Luc-3'UTR). “An” indicates the 
presence of a poly(A) tail. The iNOS 3'-UTR contains AREs (AUUU(U)A $\times 6$ ), which contribute to mRNA stabilization. (B, C) Each construct was introduced into hepatocytes, and the cells were treated with IL-1 $\beta(1 \mathrm{nM})$ in the presence or absence of metformin ( $500 \mu \mathrm{g} / \mathrm{ml})$ for $8 \mathrm{~h}$ for pRiNOS-Luc-SVpA (B) and $5 \mathrm{~h}$ for pRiNOS-Luc-3'UTR (C). Luciferase activity was normalized by $\beta$-galactosidase activity. Fold activation was calculated by dividing luciferase activity by control activity (without IL-1 $\beta$ and metformin). Data are presented as the mean \pm SD for $n=4$ dishes. $* \mathrm{P}<0.05$ versus IL-1 $\beta$ alone. (D) The cells were treated with IL-1 $\beta(1 \mathrm{nM})$ in the presence or absence of metformin $(500 \mu \mathrm{g} / \mathrm{ml})$ for the indicated times. Total RNA was analyzed by strand-specific RT-PCR to detect the iNOS gene antisense transcript (asRNA).

\section{Metformin has no effect on NF-אB activation but it inhibits IL-1RI upregulation.}

There are two essential signaling pathways for induction of iNOS, called the IKB kinase and phosphatidylinositol 3-kinase (PI3K)/Akt pathways. In the former pathway, IL-1 $\beta$ stimulates

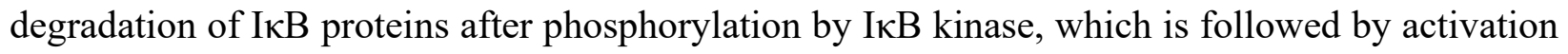
of NF- $\mathrm{KB}$ (i.e., translocation from the cytoplasm to the nucleus and DNA binding). An electrophoretic mobility shift assay with nuclear extracts demonstrated that metformin did not inhibit NF- $\mathrm{kB}$ activation at $1-5 \mathrm{~h}$, which indicated no blockade of NF- $\mathrm{kB}$ nuclear translocation (Fig. 5). Metformin also had no effect on IkB degradation (data not shown).

In the PI3K/Akt pathway, IL-1 $\beta$ stimulates upregulation of IL-1RI by activation of PI3K/Akt $[30,31]$. Immunoprecipitation-western blotting analysis demonstrated that metformin inhibited phosphorylation (activation) of Akt, a downstream kinase of PI3K (Fig. 6A). Real-time RT-PCR and western blotting analyses revealed that metformin reduced IL-1RI mRNA and protein expression levels (Fig. 6B and 6C).

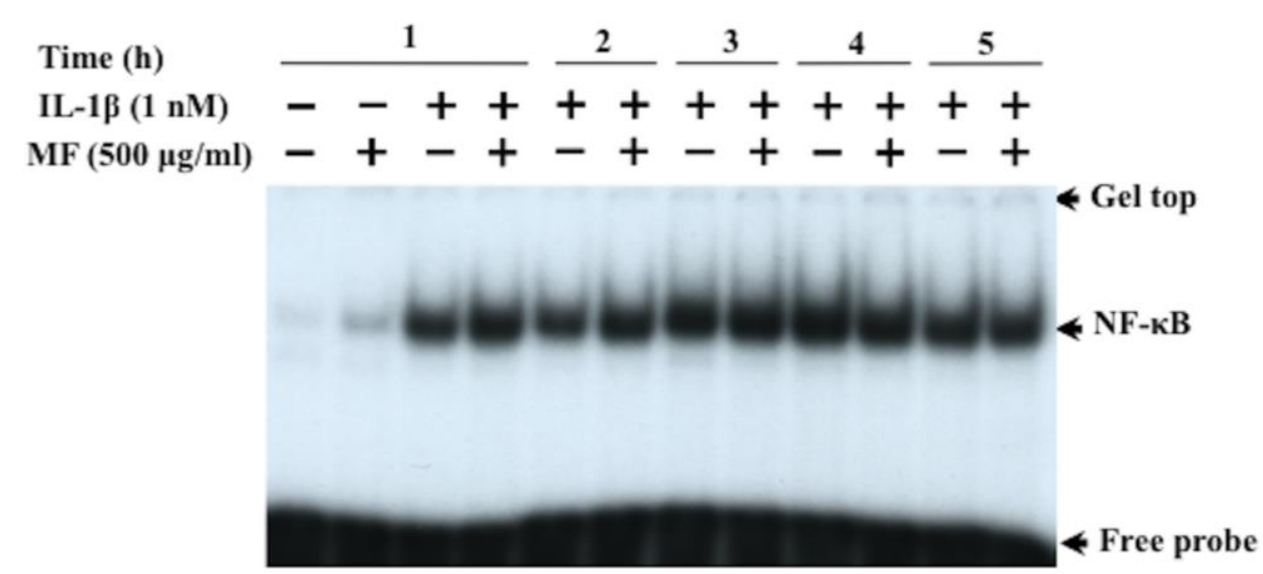

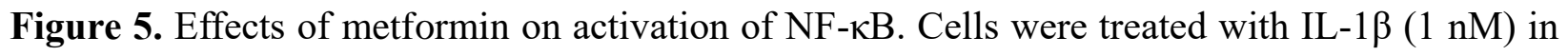
the presence or absence of metformin $(500 \mu \mathrm{g} / \mathrm{ml})$ for the indicated times. Activation of NF- $\mathrm{kB}$. Nuclear extracts ( $4 \mu \mathrm{g}$ of protein) were analyzed using an electrophoretic mobility shift assay. Representative results of three independent experiments are shown. 
A
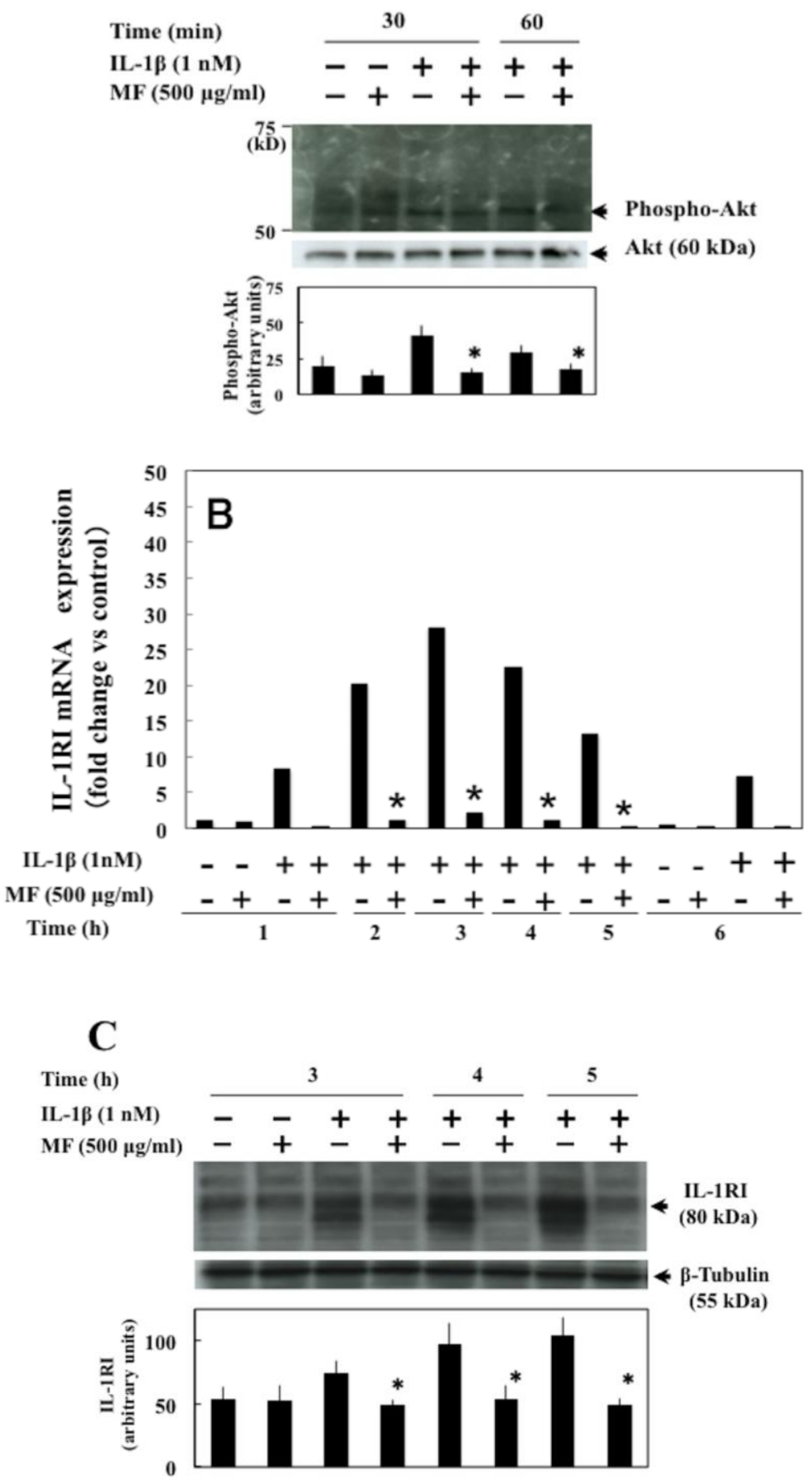

Figure 6. Effects of metformin on upregulation of type I IL-1 receptor. Cells were treated with IL$1 \beta(1 \mathrm{nM})$ in the presence or absence of metformin $(500 \mu \mathrm{g} / \mathrm{ml})$ for the indicated times. (A) Phosphorylation of Akt. Total cell lysates were immunoprecipitated with an anti-Akt antibody, followed by immunoblotting (SDS-PAGE in a gel with a gradient of 6-9\%) with an anti-phospho-Akt or anti-Akt antibody. (B) Total RNA was analyzed using strand-specific RT-PCR to detect type I IL-1 receptor (IL-1RI) mRNA, using EF mRNA as an internal control. (C) Cell lysates (50 $\mu \mathrm{g}$ of protein) were subjected to SDS-PAGE in a 7.5\% gel, and immunoblotted with an anti-IL-1RI or anti- $\beta$-tubulin antibody. The bands corresponding to phospho-Akt or IL-1RI were quantitated by densitometry (lower; mean $\pm \mathrm{SD} ; \mathrm{n}=3$ experiments). ${ }^{*} \mathrm{P}<0.05$ versus IL- $1 \beta$ alone 


\section{Delayed administration of metformin inhibits iNOS induction.}

We examined whether delayed administration of metformin affects induction of iNOS. Metformin was added to the medium $0-5 \mathrm{~h}$ after the addition of IL-1 $\beta$. Delayed administration of metformin up to $2 \mathrm{~h}$ after IL-1 $\beta$ addition still markedly inhibited NO production, although the magnitude of inhibition decreased in a time-dependent manner (Fig. 7).

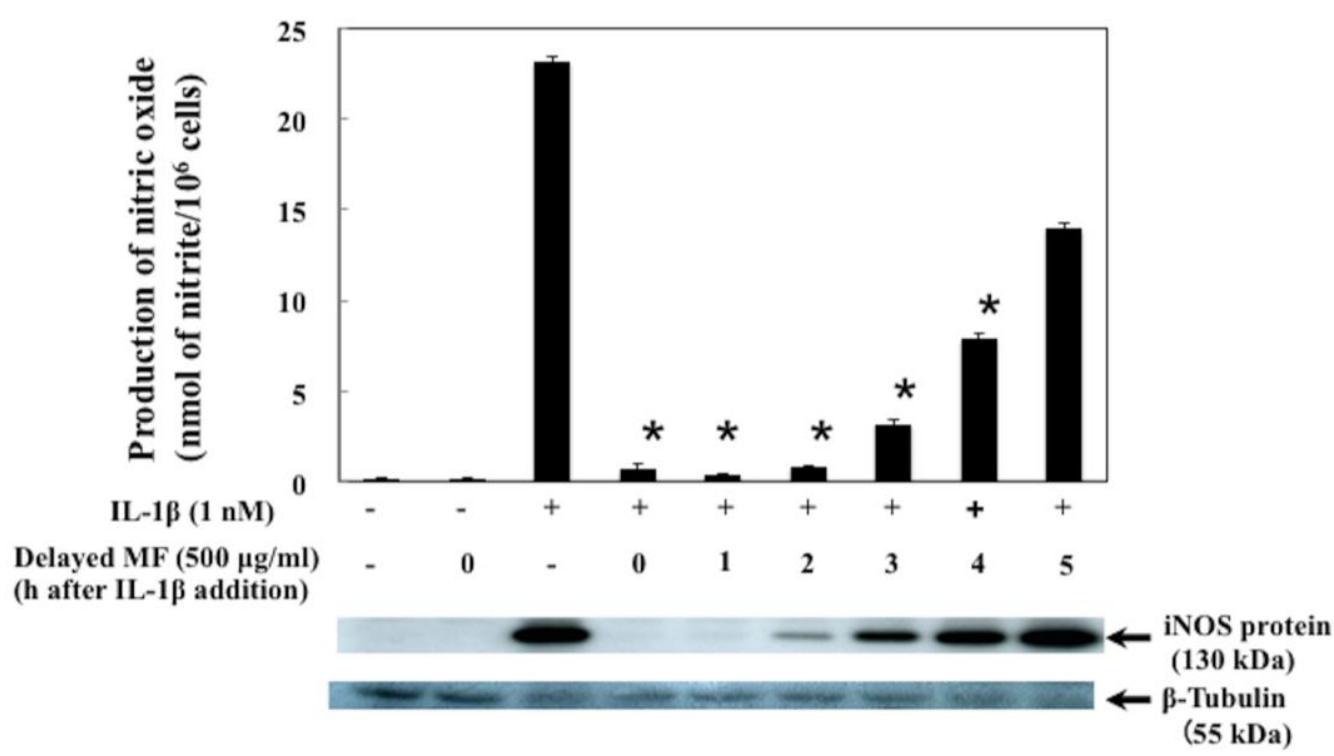

Figure 7. Effects of delayed metformin administration on induction of iNOS in hepatocytes. Cells were treated with metformin $(500 \mu \mathrm{g} / \mathrm{ml})$ at $0-4 \mathrm{~h}$ after addition of IL-1 $\beta(1 \mathrm{nM})$. The effects of metformin on NO production (upper panel) and iNOS protein (lower panel) were analyzed at $8 \mathrm{~h}$ after addition of IL-1 $\beta$. Nitrite levels were measured in the culture medium. Data are presented as the mean $\pm \mathrm{SD}, \mathrm{n}=3$ dishes/point; $* \mathrm{P}<0.05$ versus IL-1 $\beta$ alone. In the western blotting panels, cell lysates (20 $\mu \mathrm{g}$ of protein) were subjected to SDS-PAGE in a $7.5 \%$ gel, and immunoblotted with an anti-iNOS or anti- $\beta$-tubulin antibody.

\section{DISCUSSION AND CONCLUSION}

In the current study, we used primary cultured rat hepatocytes stimulated by the proinflammatory cytokine IL-1 $\beta$ (in vitro liver injury model) [10] to investigate the effects and mechanisms of metformin in the injured liver. We found that metformin inhibited the induction of iNOS and its mRNA and protein expression, thereby preventing NO production [Fig. 1A, 1B and 1C]. Additionally, in transfection experiments with iNOS promoter constructs (containing two NF- $\mathrm{B}$ binding sites, Fig. 4A), metformin decreased iNOS mRNA expression by inhibiting its mRNA synthesis and stabilization steps (Fig. 4B and 4C). For iNOS mRNA stabilization, metformin decreased iNOS asRNA expression (Fig. 4D). We recently reported that asRNAs are often 
transcribed from many inducible genes, such as iNOS and TNF- $\alpha$ [25]. The iNOS asRNA interacted with and stabilized iNOS mRNA [32,33].

Proinflammatory cytokines, such as TNF- $\alpha$, IL-1 $\beta$, and IL-6, play an important role in injury to multiple organs in addition to the liver. Metformin also inhibited mRNA expression of TNF- $\alpha$, CINC-1, and IL-6 (Fig. 3A, 3B, and 3C) and conversely enhanced mRNA expression of the antiinflammatory cytokine IL-10 (Fig. 3D). Results suggested that metformin markedly inhibited the inflammatory pathway.

Metformin had no effect on NF- $\kappa$ B activation in the EMSA experiment (Fig. 5). Conversely, we found that metformin inhibited IL-1RI upregulation (Fig. 6B and 6C) by reducing Akt phosphorylation (Fig. 6A). Isoda et al. reported that metformin demonstrated anti-inflammatory effects by sustaining NF- $\kappa$ B activity through the PI3K/AKT pathway in human vascular wall cells [34]. Because transfection experiments with iNOS promoter luc-constructs containing two NF- $\kappa \mathrm{B}$ binding sites (Fig. $4 \mathrm{~B}$ and 4C) demonstrated that metformin inhibited the activities of both constructs, we cannot rule out the possibility that metformin inhibits the promoter transactivation through NF- $\kappa \mathrm{B}$. It is known that a variety of inflammatory genes including TNF- $\alpha$ and CINC- 1 is regulated by transcription factor NF- $\kappa \mathrm{B}$. NF- $\kappa \mathrm{B}$ might be involved in mechanisms of the decreased mRNA expression of these genes.

Delayed metformin treatment after IL- $1 \beta$ addition also caused a marked reduction in iNOS induction and NO production (Fig. 7). This observation may be of clinical importance because initiation of therapeutic metformin treatment is usually delayed from the onset of disease. Liver steatosis induced by a high fat diet is associated with impaired insulin-induced liver vasodilation. These findings were documented after only 3 days of high fat diet administration, which is enough to induce liver steatosis and to impair insulin signaling, fibrosis, or other features of advanced NAFLD [35]. Appropriate regulation of inflammatory reactions is essential in preventing the onset of organ damage and early recovery.

Many studies, ranging from basic to clinical studies, have been conducted on metformin and its clinical applications in treating various diseases [37-41]. Recent reports suggest that metformin may have the therapeutic potential to treat liver injury $[42,43]$, in addition to its efficacy in treating type 2 diabetes [44]. Our results demonstrate that metformin may have anti-inflammatory effects in the liver. To link our results with previous work on metformin in the diabetic context, we speculate that metformin may block the inflammatory pathway associated with a wide variety of liver injury. Based on the range of metformin's effects, metformin itself may be useful in treating and preventing some diseases, but fully establishing its potential will require further studies and clinical applications.

Metformin can prevent IL-1 $\beta$-stimulated liver injury in cultured hepatocytes by inhibiting induction of inflammatory mediators such as iNOS and TNF- $\alpha$. Metformin may have a therapeutic potential to treat liver injury. However, it is reported that metformin should be used with caution 
for patients with liver injury clinically. Consequently, metformin may have a therapeutic potential to treat chronic liver injury in NASH rather than acute liver injury.

List of Abbreviations: MF, metformin; iNOS, inducible nitric oxide synthase; TNF- $\alpha$, tumor necrosis factor-alpha; NO, nitric oxide ; IL, interleukin; NF-кB, nuclear factor-kappa B; CINC-1, cytokine-induced neutrophil chemoattractant-1; DMSO, dimethyl sulfoxide; LDH, lactate dehydrogenase; SDS-PAGE, sodium dodecyl sulfate-polyacrylamide gel electrophoresis; IL-1RI, the type I interleukin-1 receptor; RT-PCR, reverse transcriptase-polymerase chain reaction.

Competing Interests: The authors declare that there are no conflicts of interest.

Authors' Contributions: RN participated in the design of the study, data collection, statistical analysis, and drafting of the manuscript. TO participated in supervision and provided oversight when the manuscript was being drafted. IH, IM, KM, YN, and MK assisted in the design of the study. MN provided advice regarding development of the protocol for the study and assisted in the design of the study.

Acknowledgements and Funding: This work was supported in part by a Grant-in-Aid for Scientific Research from the Ministry of Education, Science, Culture and Sports of Japan, and by grants from the Science Research Promotion Fund of the Japan Private School Promotion Foundation.

\section{REFERENCES}

1. Madiraju AK, Erion DM, Rahimi Y, Zhang XM, Braddock DT, et al.: Metformin suppresses gluconeogenesis by inhibiting mitochondrial glycerophosphate dehydrogenase. Nature 2014, 510: 542-546.

2. Zhou G, Myers R, Li Y, Chen Y, Shen X, et al.: Role of AMP-activated protein kinase in mechanism of metformin action. J Clin Invest 2001, 108: 1167-1174

3. Sanyal AJ: Mechanisms of Disease: pathogenesis of nonalcoholic fatty liver disease. Nat Clin Pract Gastroenterol Hepatol 2005, 2: 46-53..

4. Sanyal AJ, Campbell-Sargent C, Mirshahi F, Rizzo WB, Contos MJ, et al.: Nonalcoholic steatohepatitis: association of insulin resistance and mitochondrial abnormalities. Gastroenterology 2001, 120: 1183-1192.

5. Ong JP, Younossi ZM: Epidemiology and natural history of NAFLD and NASH. Clin Liver Dis 2007, 11: 1-16, vii.

6. Angulo P: GI epidemiology: nonalcoholic fatty liver disease. Aliment Pharmacol Ther 2007, 25: 883-889. 
7. Colasanti M, Suzuki H: The dual personality of NO. Trends in Pharmacological Sciences 2000, 21: 249-252.

8. Mantena SK, Vaughn DP, Andringa KK, Eccleston HB, King AL, et al.: High fat diet induces dysregulation of hepatic oxygen gradients and mitochondrial function in vivo. Biochem J 2009, 417: 183-193.

9. Raso GM, Esposito E, Iacono A, Pacilio M, Cuzzocrea S, et al.: Comparative therapeutic effects of metformin and vitamin $\mathrm{E}$ in a model of non-alcoholic steatohepatitis in the young rat. Eur J Pharmacol 2009, 604: 125-131.

10. Kaibori M, Okumura T, Sato K, Nishizawa M, Kon M: Inducible Nitric Oxide Synthase Expression in Liver Injury: Liver Protective Effects on Primary Rat Hepatocytes. Inflamm Allergy Drug Targets 2015, 14: 77-83.

11. Tsuchiya H, Kaibori M, Yanagida H, Yokoigawa N, Kwon AH, et al.: Pirfenidone prevents endotoxin-induced liver injury after partial hepatectomy in rats. Journal of Hepatology 2004, 40: 94-101.

12. Tsuji K, Kwon AH, Yoshida H, Qiu Z, Kaibori M, et al.: Free radical scavenger (edaravone) prevents endotoxin-induced liver injury after partial hepatectomy in rats. Journal of Hepatology 2005, 42: 94-101.

13. Tanaka H, Uchida Y, Kaibori M, Hijikawa T, Ishizaki M, et al.: $\mathrm{Na}+\mathrm{H}+$ exchanger inhibitor, FR183998, has protective effect in lethal acute liver failure and prevents iNOS induction in rats. Journal of Hepatology 2008, 48: 289-299.

14. Ishizaki M, Kaibori M, Uchida Y, Hijikawa T, Tanaka H, et al.: Protective effect of FR183998, a Na+/H+ exchanger inhibitor, and its inhibition of iNOS induction in hepatic ischemia-reperfusion injury in rats. Shock 2008, 30: 311-317.

15. Hijikawa T, Kaibori M, Uchida Y, Yamada M, Matsui K, et al.: Insulin-like growth factor 1 prevents liver injury through the inhibition of TNF-alpha and iNOS induction in Dgalactosamine and LPS-treated rats. Shock 2008, 29: 740-747.

16. Nakanishi H, Kaibori M, Teshima S, Yoshida H, Kwon AH, et al.: Pirfenidone inhibits the induction of iNOS stimulated by interleukin-1beta at a step of NF-kappaB DNA binding in hepatocytes. J Hepatol 2004, 41: 730-736.

17. Yoshida H, Kwon AH, Kaibori M, Tsuji K, Habara K, et al.: Edaravone prevents NOS expression by inhibiting its promoter transactivation and mRNA stability in cytokinestimulated hepatocytes. Nitric Oxide-Biology and Chemistry 2008, 18: 105-112.

18. Yuan H, Li L, Zheng W, Wan J, Ge P, et al.: Antidiabetic drug metformin alleviates endotoxin-induced fulminant liver injury in mice. Int Immunopharmacol 2012, 12: 682688. 
19. Kato Y, Koide N, Komatsu T, Tumurkhuu G, Dagvadorj J, et al.: Metformin attenuates production of nitric oxide in response to lipopolysaccharide by inhibiting MyD88independent pathway. Horm Metab Res 2010, 42: 632-636.

20. Nath N, Khan M, Paintlia MK, Singh I, Hoda MN, et al.: Metformin attenuated the autoimmune disease of the central nervous system in animal models of multiple sclerosis. J Immunol 2009, 182: 8005-8014.

21. Seglen PO: Preparation of isolated rat liver cells. Methods Cell Biol 1976, 13: 29-83.

22. Horiuti Y, Ogishima M, Yano K, Shibuya Y: Quantification of cell nuclei isolated from hepatocytes by cell lysis with nonionic detergent in citric acid. Cell Struct Funct 1991, 16: 203-207.

23. Green LC, Wagner DA, Glogowski J, Skipper PL, Wishnok JS, et al.: Analysis of nitrate, nitrite, and [15N]nitrate in biological fluids. Anal Biochem 1982, 126: 131-138.

24. Chomczynski P, Sacchi N: Single-step method of RNA isolation by acid guanidinium thiocyanate-phenol-chloroform extraction. Anal Biochem 1987, 162: 156-159.

25. Oda M, Sakitani K, Kaibori M, Inoue T, Kamiyama Y, et al.: Vicinal dithiol-binding agent, phenylarsine oxide, inhibits inducible nitric-oxide synthase gene expression at a step of nuclear factor-kappaB DNA binding in hepatocytes. J Biol Chem 2000, 275: 4369-4373.

26. Nishizawa M, Ikeya Y, Okumura T, Kimura T: Post-transcriptional inducible gene regulation by natural antisense RNA. Front Biosci 2015; 20(1): 1-39.

27. Bradford MM: A rapid and sensitive method for the quantitation of microgram quantities of protein utilizing the principle of protein-dye binding. Anal Biochem 1976, 72: 248254.

28. Matsui K, Kawaguchi Y, Ozaki T, Tokuhara K, Tanaka H, et al.: Effect of active hexose correlated compound on the production of nitric oxide in hepatocytes. JPEN J Parenter Enteral Nutr 2007, 31: 373-380; discussion 380-371.

29. Kleinert H, Pautz A, Linker K, Schwarz PM: Regulation of the expression of inducible nitric oxide synthase. Eur J Pharmacol 2004, 500: 255-266.

30. Tanaka H, Uchida Y, Kaibori M, Hijikawa T, Ishizaki M, et al.: $\mathrm{Na}+\mathrm{H}+$ exchanger inhibitor, FR183998, has protective effect in lethal acute liver failure and prevents iNOS induction in rats. Journal of Hepatology 2008, 48: 289-299.

31. Teshima S, Nakanishi H, Nishizawa M, Kitagawa K, Kaibori M, et al.: Up-regulation of IL-1 receptor through PI3K/Akt is essential for the induction of iNOS gene expression in hepatocytes. J Hepatol 2004, 40: 616-623.

32. Matsui K, Nishizawa M, Ozaki T, Kimura T, Hashimoto I, et al.: Natural antisense transcript stabilizes inducible nitric oxide synthase messenger RNA in rat hepatocytes. Hepatology 2008, 47: 686-697. 
33. Yoshigai E, Hara T, Araki Y, Tanaka Y, Oishi M, et al.: Natural antisense transcripttargeted regulation of inducible nitric oxide synthase mRNA levels. Nitric OxideBiology and Chemistry 2013, 30: 9-16.

34. Isoda K, Young JL, Zirlik A, MacFarlane LA, Tsuboi N, et al.: Metformin inhibits proinflammatory responses and nuclear factor-kappaB in human vascular wall cells. Arterioscler Thromb Vasc Biol 2006, 26: 611-617.

35. Samuel VT, Liu ZX, Qu X, Elder BD, Bilz S, et al.: Mechanism of hepatic insulin resistance in non-alcoholic fatty liver disease. J Biol Chem 2004, 279: 32345-32353.

36. Ghazeeri GS, Nassar AH, Younes Z, Awwad JT: Pregnancy outcomes and the effect of metformin treatment in women with polycystic ovary syndrome: an overview. Acta Obstet Gynecol Scand 2012, 91: 658-678.

37. Feng W, Gao C, Bi Y, Wu M, Li P, et al.: Randomized trial comparing the effects of gliclazide, liraglutide, and metformin on diabetes with non-alcoholic fatty liver disease. J Diabetes 2017, 9: 800-809.

38. Marchesini G, Brizi M, Bianchi G, Tomassetti S, Zoli M, et al.: Metformin in nonalcoholic steatohepatitis. Lancet 2001, 358: 893-894.

39. Yuan H, Li L, Zheng W, Wan J, Ge P, et al.: Antidiabetic drug metformin alleviates endotoxin-induced fulminant liver injury in mice. Int Immunopharmacol 2012, 12: 682688.

40. Masich A, Badowski ME, Liedtke MD, Fulco PP: Evaluation of the concurrent use of dolutegravir and metformin in human immunodeficiency virus-infected patients. Int $\mathrm{J}$ STD AIDS 2017, 28: 1229-1233.

41. Driscoll SD, Meininger GE, Ljungquist K, Hadigan C, Torriani M, et al.: Differential effects of metformin and exercise on muscle adiposity and metabolic indices in human immunodeficiency virus-infected patients. J Clin Endocrinol Metab 2004, 89: 2171-2178.

42. Kalariya NM, Shoeb M, Ansari NH, Srivastava SK, Ramana KV: Antidiabetic drug metformin suppresses endotoxin-induced uveitis in rats. Invest Ophthalmol Vis Sci 2012, 53: $3431-3440$.

43. Volarevic V, Misirkic M, Vucicevic L, Paunovic V, Simovic Markovic B, et al.: Metformin aggravates immune-mediated liver injury in mice. Arch Toxicol 2015, 89: 437-450.

44. Pasarín M, Abraldes JG, Rodríguez-Vilarrupla A, La Mura V, García-Pagán JC, et al.: Insulin resistance and liver microcirculation in a rat model of early NAFLD. Journal of Hepatology 2011, 55: 1095-1102. 